\title{
The Long-Term Outcome of CT-Guided Pulsed Radiofrequency in the Treatment of Idiopathic Glossopharyngeal Neuralgia: A Retrospective Multi-Center Case Series
}

This article was published in the following Dove Press journal:

Journal of Pain Research

\author{
Yitong Jia ${ }^{1, *}$ \\ Niti Shrestha $\mathbb{( D}^{2, *}$ \\ Xiaodi Wang $\mathbb{1 D}^{2, *}$ \\ Tao Wang ${ }^{3}$ \\ Fang Luo ${ }^{2}$ \\ 'Department of Anesthesiology, Xuanwu \\ Hospital, Capital Medical University, \\ Beijing, People's Republic of China; \\ ${ }^{2}$ Department of Pain Management, Beijing \\ Tiantan Hospital, Capital Medical \\ University, Beijing, People's Republic of \\ China; ${ }^{3}$ Department of Neurosurgery, \\ Beijing Tiantan Hospital, Capital Medical \\ University, Beijing, People's Republic of \\ China
}

*These authors contributed equally to this work
Correspondence: Fang Luo

Department of Pain Management, Beijing

Tiantan Hospital, Capital Medical

University, Beijing, People's Republic of

China

Email136I|326978@I63.com
Background: Safer and minimal invasive treatment options with minor side effects are in great demand in the treatment of glossopharyngeal neuralgia (GPN). Pulsed radiofrequency (PRF) is a micro-destructive procedure that could be applied repeatedly without irreversible damage to target tissue. However, few studies have reported the long-term clinical outcomes of PRF in the management of idiopathic GPN patients.

Methods: We retrospectively investigated the efficacy and safety of computed tomography (CT)-guided PRF in the treatment of 30 patients with idiopathic GPN in a multi-center clinical study. Numeric rating scale (NRS) score was used to evaluate pain intensity before and after PRF treatment. The effective rate was defined as the percentage of patients with NRS reduction of more than $50 \%$. Baseline characteristics, surgical records, initial pain relief, time to take effect, long-term outcomes, patient satisfaction using a five-level Likert Scale, the incidence of recurrence as well as subsequent treatment choices, intraoperative and postoperative complications were retrieved from electronic medical records.

Results: A total of 30 idiopathic GPN patients who received PRF under CT-guidance were included in our study and the initial effective rate was $93.3 \%$. The cumulative proportion of patients with satisfactory pain relief survival was $93.3 \%$ at 12 months, $89.6 \%$ at 24 months, $85.3 \%$ at 36 months, $79.6 \%$ at 48 months, $73.0 \%$ at 60 months and 72 months, and $54.8 \%$ at 84 months, 108 months as well as 120 months. No serious morbidity or mortality were observed in any of the cases. The median patient satisfaction in Likert scale rating was 4.0 (IQR, 3.0-5.0)

Conclusion: According to our results, PRF is an effective and safe therapy for patients with idiopathic GPN. This minimally invasive, micro-destructive, neuro-modulatory technique could be a potential intervention of choice for the treatment of GPN patients who respond poorly to pharmacological treatment.

Keywords: pulsed radiofrequency, idiopathic glossopharyngeal neuralgia, long-term outcome, efficacy, safety, retrospective study

\section{Background}

Glossopharyngeal neuralgia (GPN) is an uncommon facial pain syndrome characterized by paroxysms of pain in the sensory distribution of the glossopharyngeal nerve (cranial nerve IX). ${ }^{1,2}$ It is reported that the crude incidence of GPN is estimated to be approximately 0.2 to 0.8 per 100,000 population per year. ${ }^{1,3,4}$ The pain is usually located in the tonsillar fossa, base of tongue, laryngeal region, 
mandibular angle and the ear. ${ }^{5}$ The characteristics of GPN are usually stabbing, sharp or shooting. ${ }^{6}$ GPN could be triggered by specific stimulus including yawning, talking, swallowing, sneezing, laughing and coughing which results in patients being afraid of talking, eating, drinking, ${ }^{6}$ sleep disturbances and may even lead to suicide. ${ }^{7}$ Moreover, GPN may also be associated with bradycardia, cardiac arrest, convulsions and syncope due to overflow of neural impulses central glossopharyngeal tracts to the central vagal tracts (solitary nucleus and the spinal trigeminal nucleus), which occurs rarely but can be lethal. ${ }^{2,6}$

Medical therapy such as carbamazepine, oxcarbazepine, gabapentin, pregabalin, and phenytoin, is the firstline of treatment for GPN patients. ${ }^{2}$ However, the therapeutic effect of these drugs is variable, and may decline over time. ${ }^{8}$ Glossopharyngeal nerve block could provide an excellent alternative to pharmacological treatment for glossopharyngeal neuralgia via injection of local anesthetic with or without steroids. However, it is difficult to obtain long-term effect even despite the guidance of ultrasound. ${ }^{9,10}$ Surgical treatments such as microvascular decompression (MVD) or glossopharyngeal nerve and upper rootlets of vagus nerve rhizotomy should be considered when patients respond poorly to conservative treatments. Several studies have demonstrated that GPN patients receiving MVD could achieve long-term painfree outcomes by isolating the offending compressing blood vessel. ${ }^{11-13}$ However, this widely used surgical treatment is still associated with several risks including dysphagia, compromised swallowing, even intracranial hemorrhage, ${ }^{14}$ and a potential for reoperation. ${ }^{5}$ Rhizotomy is considered as a safe and useful alternative when exploratory surgery cannot identify a vascular conflict. ${ }^{15,16}$ Nevertheless, complications such as sensory deficits, dysphagia and vocal cord paralysis could be associated with rhizotomy. ${ }^{6,17}$ Gamma knife radiosurgery ${ }^{18}$ (GKRS) has been proposed as an effective treatment option for GPN without permanent complications. However, it is reported that the failure ratio of GKRS within a mean follow-up duration of 24.6 months could increase up to $30 \% .{ }^{19}$ Percutaneous radiofrequency thermocoagulation has been successfully applied in the treatment of idiopathic and secondary GPN, however, it has been associated with several complications including dysphagia, dysesthesias, and diminished gag reflex. ${ }^{5,20}$ Therefore, a safer and minimal invasive treatment option with minor side effects is in great demand for GPN.
Pulsed radiofrequency (PRF) is a micro-destructive procedure which applies short pulse of radiofrequency to ganglion or peripheral nerve, and has neuromodulatory effect in the treatment of neuropathic pain. ${ }^{21}$ PRF applies high-frequency current in a pulse of 20 milliseconds, every 500 milliseconds. $^{22}$ The heat generated in each active cycle could dissipate in the long resting phase of 480 milliseconds, so that the temperature does not exceed $42{ }^{\circ} \mathrm{C} .{ }^{23}$ Unlike radiofrequency thermocoagulation, which selectively destroys pain fibers via continuous radiofrequency with the temperature over $65^{\circ} \mathrm{C}^{1}$ PRF could be applied repeatedly for several times without irreversible damage to neuronal tissue. Shah et $\mathrm{al}^{24}$ presented the first case of GPN (chronic post-tonsillectomy pain) treated with PRF which attained satisfactory pain relief in 2003. In 2005, Abejón et $\mathrm{al}^{25}$ performed PRF on 1 idiopathic GPN and 1 secondary GPN, in which the results revealed that the treatment was effective for both patients for over 6 months. Bharti et $\mathrm{al}^{26}$ also showed the efficacy and safety of PRF on the treatment of GPN secondary to oropharyngeal cancer in a clinical trial with 25 sample sizes, and demonstrated that PRF was a beneficial treatment option for secondary GPN patients without serious complications. However, there is a scarcity of studies evaluating the longterm clinical outcomes of PRF in the management of idiopathic GPN patients. In this multicenter retrospective study, we intend to determine the long-term efficacy and safety of PRF for the treatment of GPN patients under the guidance of computed tomography (CT).

\section{Methods}

\section{Patients}

This retrospective study has obtained approval from the Institutional Review Board of Beijing Tiantan Hospital affiliated to Capital Medical University (No. ky 2020-01702). The patient data accessed in this study complied with relevant data protection and privacy regulations. We collected the records of 30 cases of idiopathic GPN patients who received PRF treatments under the guidance of $\mathrm{CT}$ at the department of pain management in Beijing Tiantan Hospital, Beijing Tiantan Puhua Hospital, and Beijing Red Cross Peace Hospital between February 2007 and October 2019. The available follow-up data ended in February 2020. Patient consent was waived due to the study's retrospective nature. Patients who fulfilled the following criteria were eligible for the study: 1) age $>18$ years; 2) diagnosed with idiopathic GPN according to the third International Classification of 
Headache Disorders (ICHD) classification; ${ }^{15,27}$ 3) patients who failed pharmacotherapy or intolerable side effects of medication; 4) undergoing PRF for GPN; 5) at least 1 month of follow-up recordings. The exclusion criteria were as follows: 1) patients with bilateral GPN; 2) patients with incomplete medical records (lack of baseline data or postoperative follow-up data).

\section{PRF Procedures}

All procedures were performed under the guidance of CT. Patients were placed in supine position with head slightly turned contralaterally on the CT scanner bed, with a thin pillow under the head. Vital parameters including noninvasive blood pressure (BP), heart rate (HR), electrocardiogram (ECG) and pulse oximetry $\left(\mathrm{SPO}_{2}\right)$ were recorded at 5-minute intervals continuously until the end of the procedure. The negative electrode of a PMG-230 Pain Management Generator (Baylis Medical Inc., Montreal, Canada) was attached to the patient's back. After sterilization, local infiltration anesthesia with $1 \%$ lidocaine was injected at a point overlying midway between the mastoid process and the angle of mandible. Then, a 21-gauge radiofrequency cannula with a 5-mm bare tip (PMF-21-100-5, Baylis Medical Inc., Montreal, Quebec, Canada) was inserted medially under CTguidance, until the needle contacted the styloid process; then, the needle was walked off the posterior portion of the styloid process and moved forward another $0.5-1.5 \mathrm{~cm}$. Intermittent CT scan (SOMATOM SIEMENS Company, Munich, Germany) was performed to confirm that the tip of the needle was localized at the medial edge of the styloid process (Figure 1). Subsequently, the needle core was removed and the plunger was withdrawn to confirm negative aspiration for blood or cerebrospinal fluid. The radiofrequency treatment electrode (PMK-21-100, Baylis Medical Inc., Montreal, Canada) was inserted into the trocar to test resistance. Sensory stimulation at $50 \mathrm{~Hz}, 0.5 \mathrm{~V}$ was performed to cause slight pain at the innervation area of glossopharyngeal nerve including the base of tongue, tonsils as well as pharynx. The direction and depth of the needle were adjusted on the basis of patients' sensations, to guarantee correct positioning. Motor stimulation at $2 \mathrm{~Hz}, 1.0 \mathrm{~V}$ was performed and the negative results indicated that no muscle
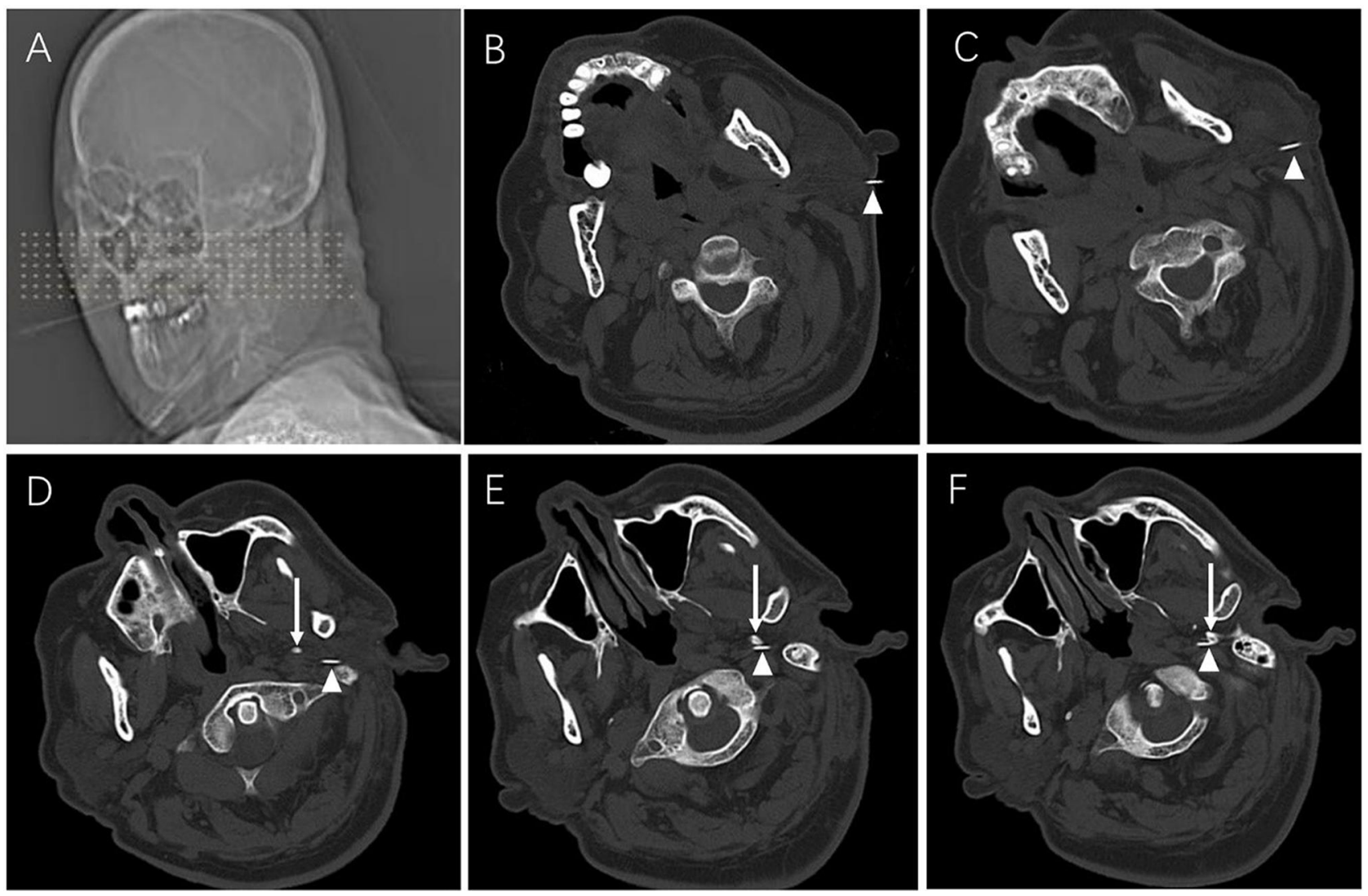

Figure I PRF procedures. (A) Location for puncture. (B-F) Consecutive CT scans showing the puncture needle gradually reaching the medial edge of styloid process. Patients were placed in a supine position with head slightly turned contralaterally. White arrows indicate the needle, white triangles indicate the styloid process. 
contraction was generated. Afterwards, PRF treatment was performed for 360 seconds. The parameters of PRF application were initiated at the voltage of $45 \mathrm{~V}$, the temperature of $42^{\circ} \mathrm{C}$, the pulse rate of $2 \mathrm{~Hz}$ at the rate of 20 milliseconds.

\section{Data Acquisition}

Baseline characteristics, surgical records and immediate efficacy were retrieved from electronic medical records of these hospitals. Long-term outcomes were acquired from a patient database that was routinely updated for medical quality management previously. The patients were followed up at 1 day, 1 week, 2 weeks, 1 month, 3 months, 6 months, and every year postoperatively. Baseline characteristics included age, gender, duration of symptoms, laterality, preoperative pain intensity, previous treatments, symptomatic site, etc.

Numeric rating scale (NRS) score ${ }^{28}$ during resting was used to evaluate pain intensity before and after PRF treatment $(0=$ no pain, $10=$ intolerable pain). The response rate was defined as cases with a reduction in pain intensity (NRS) of more than $50 \%$ per total number of cases $* 100 \%$. The initial effective rate was defined as the percentage of patients who attained effective pain relief within one month postoperatively. Patient satisfaction was assessed using five-level Likert Scale (1-5) (Table 1). ${ }^{29}$ Consumption of analgesics (pre-operation and postoperation), time to take effect, incidence of recurrence and subsequent treatment choices were also recorded.

Intraoperative complications included hypotension and bradycardia. Other complications including dysphagia, lingual numbness and abnormal sensation, pharynx and larynx numbness, hoarseness, abnormal sense of taste were also collected. The classification by Landriel Ibanez and associates was used to evaluate the severity of complications in our study (Table 2). ${ }^{30}$

\section{Statistical Analysis}

Statistical analyses were performed using IBM SPSS Statistics software (Version 26, IBM Inc., USA). The Kolmogorov-Smirnov test was used to assess normality

Table I Likert Scale

\begin{tabular}{|l|l|}
\hline Likert Scale & Explanations \\
\hline 1 & Very unsatisfied \\
2 & Unsatisfied \\
3 & Neutral \\
4 & Satisfied \\
5 & Very satisfied \\
\hline
\end{tabular}

Table 2 The Landriel Ibanez Classification of Complications

\begin{tabular}{|l|l|}
\hline Grade & Explanations \\
\hline Grade I & $\begin{array}{l}\text { Non-life-threatening complications requiring no invasive } \\
\text { treatment }\end{array}$ \\
Grade II & Complications requiring invasive treatments \\
Grade III & Life-threatening complications requiring management in ICU \\
Grade IV & Complication resulting in death \\
\hline
\end{tabular}

distribution of patient data. Normally distributed continuous data were presented as mean with standard deviation (SD) while non-normally distributed continuous data and ordinal data were described as median with interquartile ranges (IQRs). Nominal data were presented as counts or percentages. Kaplan-Meier curves were used to describe response rate after PRF treatment. A P value $<0.05$ was considered statistically significant.

\section{Results \\ Demographics}

Between February 2007 and October 2019, a total of 30 idiopathic GPN patients received PRF under CT-guidance, in the department of pain management of Beijing Tiantan Hospital, Beijing Tiantan Puhua Hospital, and Beijing Red Cross Peace Hospital. Follow-up duration ranged from 1 month to 120 months and the median follow-up period was 42.5 months. The demographic data and baseline characteristics are presented in Table 3. The mean age of GPN patients was $62.2 \pm 12.6$ years, the maximum age was 84 years, while the minimum age was 36 years. The mean age at onset of GPN patients was $55.1 \pm 16.2$ years, the maximum age was 83 years, while the minimum age of onset was 20 years. The median duration of disease was 60 (IQR, 24.0-96.0) months while the median follow-up duration was 42.5 (IQR,24-61.5) months. Of the 30 patients, $11(36.7 \%)$ were male, and $12(40.0 \%)$ had GPN on the right side. In 22 cases, patients experienced tongue, pharynx, or larynx pain, 3 patients presented with symptoms in the ear and 12 cases presented with pain below the angle of mandible. The mean prior carbamazepine consumption was 500 (IQR, 300-600) mg. All GPN patients in our study responded poorly to oral medications, with a median NRS score of 7 (IQR, 6-8).

\section{Initial Pain Relief}

The median NRS score, immediately after the procedure was 4 (IQR, 3-5), which was significantly lower than that before the operation $(\mathrm{P}<0.05)$. Regarding initial pain relief, 
Table 3 The Demographic Data and Baseline Characteristics of GPN Patients

\begin{tabular}{|c|c|}
\hline Characteristics & $\begin{array}{l}\text { Mean } \pm \text { SD, N(\%) or } \\
\text { Median (IQR) }\end{array}$ \\
\hline No. of patients & 30 \\
\hline Age (years, mean $\pm S D$ ) & $62.2 \pm 12.6$ \\
\hline Age at onset (years, mean $\pm S D$ ) & $55.1 \pm 16.2$ \\
\hline $\begin{array}{l}\text { Duration of disease [months, Median } \\
(\mathrm{IQR})]\end{array}$ & $60(24.0-96.0)$ \\
\hline $\begin{array}{l}\text { Follow-up duration [months, Median } \\
(\mathrm{IQR}) \text { ] }\end{array}$ & $42.5(24,61.5)$ \\
\hline Sex (male, \%) & II (36.7\%) \\
\hline Laterality (right, \%) & $12(40.0 \%)$ \\
\hline Symptomatic site & $\mathrm{n}(\%)$ \\
\hline Tongue, pharynx, or larynx & $22(73.3 \%)$ \\
\hline Auricular pain & $3(10 \%)$ \\
\hline Beneath the angle of the lower jaw & $12(40.0 \%)$ \\
\hline $\begin{array}{l}\text { Prior carbamazepine consumption (mg, } \\
\text { mean } \pm S D)\end{array}$ & $473.3 \pm 152.9$ \\
\hline $\begin{array}{l}\text { Preoperative numeric rating scale } \\
{[0-10, \text { Median }(\mathrm{IQR})]}\end{array}$ & $7(6-8)$ \\
\hline
\end{tabular}

28 GPN patients experienced effective pain relief within one month postoperatively and the initial effective rate was $93.3 \%$. The median time to take effect was 3 (IQR,2-5; range,1-14) days. The carbamazepine consumption at one month after treatment was 200 (IQR,100-200) $\mathrm{mg}$, which was significantly lower than prior carbamazepine consumption $(\mathrm{P}<0.05)$. Two cases responded poorly to PRF even with oral carbamazepine; one of which had a family history of GPN and the other one had a rather long GPN history of 120 months. Both the 2 ineffective cases chose to receive MVD one month after PRF treatment.

\section{Long-Term Outcomes}

Among 30 patients, 23 patients experienced satisfactory pain relief with or without oral medications throughout the entire follow-up period. The cumulative proportion of recurrence-free survival is shown as Kaplan-Meier curve (Figure 2). The cumulative proportion of patients with satisfactory pain relief survival was $93.3 \%$ at 12 months, $89.6 \%$ at 24 months, $85.3 \%$ at 36 months, $79.6 \%$ at 48 months, $73.0 \%$ at 60 months and 72 months, and $54.8 \%$ at 84 months, 108 months as well as 120 months. Five patients suffered from relapse of GPN symptoms at $13,33,51,60$, and 84 months after PRF treatment (Figure 3). Among the 5 recurrence cases; 2 patients received repeated PRF, 2 patients

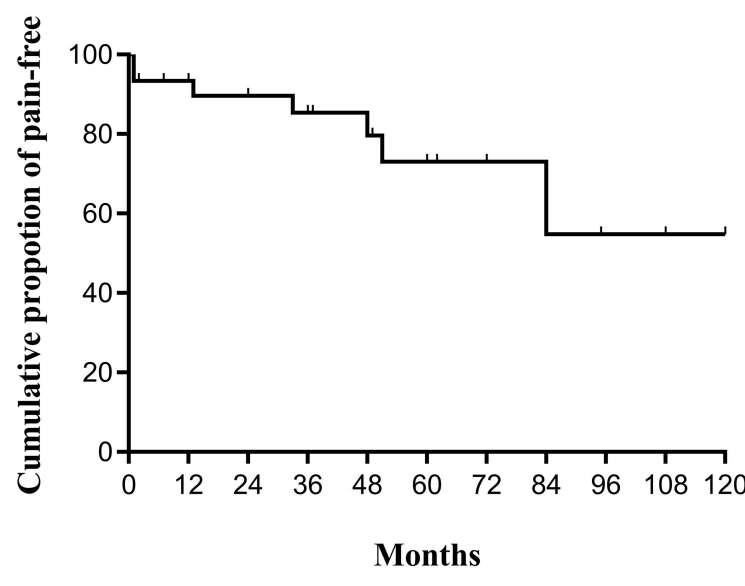

No. at Risk $\begin{array}{lllllllllll}30 & 26 & 24 & 20 & 15 & 11 & 7 & 4 & 3 & 2 & 1\end{array}$

Figure 2 Kaplan-Meier recurrence-free survival curves for idiopathic glossopharyngeal neuralgia patients who underwent CT-guided pulsed radiofrequency.

chose to undergo radiofrequency thermocoagulation, and 1 patient underwent MVD. All relapsed patients attained complete pain relief, regardless of the treatment they decided to undergo. For the 2 patients who chose PRF treatment for the second time, the follow-up period for one patient was 7 months and the other patient was 24 months. No pain recurrence was observed during the second follow-up duration.

\section{Complications}

Intraoperative and postoperative complications are shown in Table 4. No serious morbidity or mortality was observed in any of the cases. During the procedure, one patient (3.3\%) developed bradycardia and the patient resumed sinus rhythm after $0.01 \mathrm{mg} / \mathrm{kg}$ atropine was administered. After the operation, 1 patient (3.3\%) had transient facial nerve neuropraxia and the symptom disappeared within 1 hour postoperatively. One patient $(3.3 \%)$ experienced dysphagia; hence, the patient's daily diet was mildly affected. The dysphagia gradually disappeared after 3 weeks. Two patients $(6.7 \%)$ developed lingual numbness and abnormal sensation, causing a slight inconvenience, as they had to chew with the contralateral side. The symptoms disappeared within 2 weeks and 3 weeks, respectively. One patient (3.3\%) experienced abnormal sense of taste, mildly impacting their dietary routines. The abnormal sense of taste improved within 3 weeks. All complications that occurred in our research were assessed as grade I, as defined by the Landriel Ibanez classification, and most patients expressed great satisfaction with a median Likert scale rating of 4.0 (IQR, 3.0-5.0). 


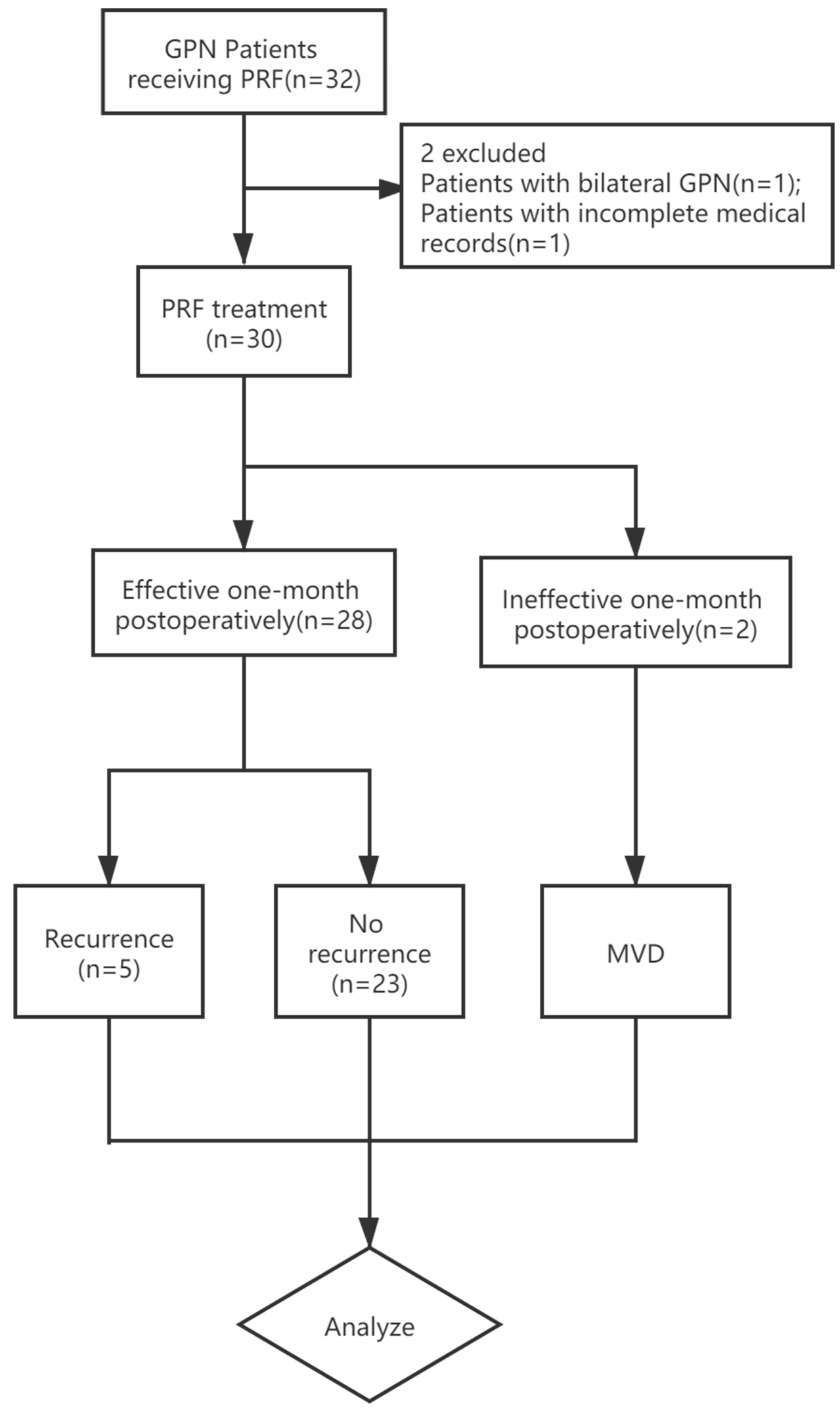

Figure 3 Flow Chart.

Abbreviations: GPN, glossopharyngeal neuralgia; PRF, pulsed radiofrequency; MVD, microvascular decompression. 
Table 4 Complications of PRF for 30 Cases

\begin{tabular}{|l|l|l|}
\hline Complications & Number & Ratio \\
\hline $\begin{array}{l}\text { Intraprocedural complications } \\
\text { Hypotension }\end{array}$ & 0 & \\
Bradycardia & $\mathrm{I}$ & $3.3 \%$ \\
\hline Postprocedural complications & & \\
Transient facial nerve neuropraxia & $\mathrm{I}$ & $3.3 \%$ \\
Dysphagia & $\mathrm{I}$ & $3.3 \%$ \\
Lingual numbness and abnormal sensation & 2 & $6.7 \%$ \\
Hoarseness & 0 & 0 \\
Abnormal sense of taste & $\mathrm{I}$ & $3.3 \%$ \\
\hline
\end{tabular}

\section{Discussion}

We evaluated the therapeutic effects of PRF in patients with idiopathic GPN and the results revealed that PRF could obtain a promising effect with an effective rate of $93.3 \%$ at 1 month postoperatively with long-term recurrence-free survival rates of $54.8 \%$ at 120 months postoperatively. The short-term efficacy we observed is consistent with that of Bharti et al, ${ }^{26}$ who reported a study about GPN secondary to oropharyngeal carcinoma that was treated by PRF. In their study, the treatment was effective in 23 out of 25 patients (92\%), for more than 3 months without major complications. However, Bharti et $\mathrm{al}^{26}$ reported that the average duration of effective pain relief was only 5 to 9 months. As a result, we speculate that PRF can attain longer duration of pain relief for the treatment of idiopathic GPN than secondary GPN. However, prospective randomized controlled trials (RCTs) are necessary to further confirm our speculation.

The efficacy of PRF is considered inferior to radiofrequency thermocoagulation for the treatment of trigeminal neuralgia (TN). ${ }^{31}$ However, there is a lack of research comparing the effectiveness of PRF and radiofrequency thermocoagulation on GPN therapy. GPN is a rare debilitating form of paroxysmal facial pain, which occurs 100 times less frequently than TN. ${ }^{7}$ As a result, the low incidence of GPN may explain the paucity of related studies. Song et al reported that the immediate pain relief rate in idiopathic GPN patients treated with radiofrequency thermocoagulation was $82.1 \%$, and long-term pain relief was $44.2 \%$ at 10 years, and $39.3 \%$ at 12.5 years postoperatively. ${ }^{5}$ Based on our research, it appears that PRF could attain greater effective rate than radiofrequency thermocoagulation treatment, probably because the definitions of observing a response in the two studies are different. In our study, the effective rate is defined as the percentage of patients with NRS reduction of more than $50 \%$ while in Song et al study, the effective rate was defined as the percentage of patients classified into Barrow Neurological Institute (BNI) class I and BNI class II outcome. Moreover, the participants included in our study were idiopathic GPN patients without any history of invasive treatments, whereas, 27 (23.1\%) patients in Song et al study underwent prior procedures such as nerve block, radiofrequency thermocoagulation, stereotactic radiosurgery or MVD. Therefore, the GPN patients in Song et al study might have been more intractable than that in our research. In addition, the treatment efficacy is perhaps associated with the technical level of pain specialists and different intraoperative imaging modalities. Prospective randomized controlled clinical trials remain to be conducted to further compare the efficacy of PRF and radiofrequency thermocoagulation, for the treatment of GPN.

There were 2 cases who did not respond to PRF treatment in our study. We assume this was probably due to the patient's family history of GPN or the patient's over a decade long history of GPN, which we suspect to be more intractable. However, whether family history or disease duration are factors influencing treatment outcomes, are yet to be studied. Recurrence could occur with any treatments, including radiofrequency thermocoagulation, ${ }^{5}$ gamma knife radiosurgery, ${ }^{18}$ and MVD; ${ }^{32} 5$ cases relapsed in our study. Up to now, the etiology of pain recurrence is not clear. Therefore, additional studies on the prevention of pain relapse are required to further improve the longterm outcomes of GPN patients.

It is worth noting that the patients in our study did not achieve excellent pain relief immediately after undergoing PRF, but rather required a median interval latency of 3 days (IQR,2-5; range,1-14) and two cases needed even more than 10 days to achieve satisfactory results. The interval latency is consistent with our previous studies on PRF for the treatment of supraorbital neuralgia, infraorbital neuralgia, and $\mathrm{TN}^{33-35} \mathrm{We}$ assume the reason for the existence of a recovery period is because PRF could cause plastic changes in pain transmission pathways and result in slow neuromodulation, which would require longer time to take effect. Therefore, after receiving PRF procedure, individual variations in posttreatment protocols should be taken into consideration, such as: adjusting oral analgesics to assist patients in getting through the recovery period without progression of pain intensity. 
In our previous study, we found that the efficacy of PRF treatment was positively correlated with the output voltage. ${ }^{36}$ Subsequently, prospective, randomized, doubleblinded studies have certified that high-voltage PRF is more effective than standard-voltage in treating idiopathic $\mathrm{TN}$ and refractory infraorbital neuralgia. ${ }^{34,37}$ However, there is a lack of research evaluating the effectiveness and safety of high-voltage PRF in the treatment of idiopathic GPN patients. Furthermore, whether the improvement of other parameters such as pulse frequency, pulse width, temperature, or treatment time could attain more satisfactory efficacy remains to be studied further.

PRF is an effective and safe method which has been applied in the treatment of diverse neuropathic pain such as $\mathrm{TN}$, occipital neuralgia, postherpetic neuralgia, and pudendal Neuralgia. ${ }^{21,38,39}$ Different from radiofrequency thermocoagulation, this method works by delivering highfrequency current to targeted nerve without heat-induced tissue damage. In this study, PRF caused less complications such as dysphagia, lingual numbness and abnormal sensation, pharynx and larynx numbness, hoarseness as well as abnormal sense of taste, compared to Song et al research, which reported the efficacy of radiofrequency thermocoagulation for GPN therapy. ${ }^{5}$ Furthermore, PRF related postoperative complications were slighter in this study, and disappeared earlier than radiofrequency thermocoagulation related complications because PRF is a treatment technology with micro-destructiveness. One patient developed bradycardia during the operation due to vagal stimulation, who immediately resumed sinus rhythm after the administration of atropine, which suggests that close hemodynamic monitoring is necessary to ensure the safety of patients during the procedure. In our study, no patient developed infection, as all procedures were performed in sanitary conditions with utmost precautionary measures. Additionally, the choice of percutaneous approach to target glossopharyngeal nerve may be related to the incidence of complications. Intraoral method is commonly performed for preemptive analgesia, and is associated with the risk of infection and may cause iatrogenic damage to several structures including vagus nerve, internal carotid artery, vertebral artery, brainstem, and upper cervical spinal nerves. ${ }^{40}$ Besides, in early years, medial part of the jugular foramen approach was used for radiofrequency treatment on GPN patients. ${ }^{41}$ However, glossopharyngeal nerve, vagus nerve and accessory nerve pass through together at the level of the jugular foramen, this increases the possibility of damaging nearby cranial nerves and internal carotid artery. ${ }^{42}$ At the styloid process level, glossopharyngeal nerve, vagus nerve and accessory nerve separate from each other, and cranial nerve IX becomes the most adjacent nerve to the distal part of the styloid process. Hence, we chose the tip of the styloid process as the target, which is consistent with Bharti et al study. ${ }^{26}$ In addition, all patients underwent CTguided treatment, in which clear visualization of the anatomic structures was ensured and neurovascular injury and puncture to adjacent structures were avoided.

\section{Limitations}

Our study has several limitations. Firstly, this is a retrospective case series report with a small sample size which unavoidably caused some selection bias. Therefore, prospective, randomized controlled researches with larger sample sizes as well as higher level of evidence are needed to further validate our outcomes. However, it will be quite difficult to conduct RCT studies due to the low incidence of GPN. Secondly, only patients with idiopathic GPN were included in our research. The long-term outcome of PRF for the treatment of secondary GPN remains to be studied further. Finally, although PRF treatment under the guidance of CT increases the rate of successful punctures and decreases the incidence of complications caused by inaccurate punctures, patients are inevitably exposed to radiation energy which may lead to safety concerns. In recent years, ultrasonography has been proven to be a safer and easier imaging modality, without ionizing radiation. ${ }^{33,43}$ Further investigations for the therapeutic efficiency of ultrasound-guided PRF operation on glossopharyngeal nerve are of great importance.

\section{Conclusions}

To the best of our knowledge, this is the first study to evaluate the long-term efficacy and safety of CT-guided PRF for the treatment of idiopathic GPN. Our results suggest that PRF is an effective and safe therapy for patients with idiopathic GPN. This minimally invasive, micro-destructive, neuromodulatory technique could be a potential intervention of choice for the treatment of GPN patients who respond poorly to pharmacological treatment.

\section{Abbreviations}

GPN, glossopharyngeal neuralgia; MVD, microvascular decompression; GKRS, gamma knife radiosurgery; PRF, pulsed radiofrequency; CT, computed tomography; BP, blood pressure; HR, heart rate; ECG, electrocardiogram; 
$\mathrm{SPO}_{2}$, pulse oximetry; NRS, Numeric rating scale; $\mathrm{SD}$, standard deviation; IQRs, interquartile ranges; RCTs, randomized controlled trials; TN, trigeminal neuralgia; BNI, Barrow Neurological Institute.

\section{Data Sharing Statement}

The datasets of the current study are available from the corresponding authors upon reasonable request.

\section{Ethics Approval and Consent to Participate}

This retrospective study has obtained approval from the Institutional Review Board of Beijing Tiantan Hospital affiliated to Capital Medical University (No. ky 2020017-02). Patient consent was waived due to the study's retrospective nature.

\section{Consent for Publication}

All authors consented.

\section{Acknowledgments}

The authors would like to thank the participants of the study for their cooperation.

\section{Author Contributions}

All authors made a significant contribution to the work reported, whether that is in the conception, study design, execution, acquisition of data, analysis and interpretation, or in all these areas; took part in drafting, revising or critically reviewing the article; gave final approval of the version to be published; have agreed on the journal to which the article has been submitted; and agree to be accountable for all aspects of the work. YJ, NS and XW contributed equally to this work. FL is responsible as corresponding author.

\section{Funding}

This study was funded and sponsored by Foundation for the Excellent Medical Staff of Beijing (No. 2014-3-035).

\section{Disclosure}

The authors report no conflicts of interest in this work.

\section{References}

1. Franzini A, Messina G, Franzini A, et al. Treatments of glossopharyngeal neuralgia: towards standard procedures. Neurol Sci. 2017;38 (Suppl 1):51-55. doi:10.1007/s10072-017-2909-6
2. Reddy GD, Viswanathan A. Trigeminal and glossopharyngeal neuralgia. Neurol Clin. 2014;32(2):539-552. doi:10.1016/j.ncl.2013. 11.008

3. Pearce JM. Glossopharyngeal neuralgia. Eur Neurol. 2006;55 (1):49-52. doi:10.1159/000091429

4. Koopman JS, Dieleman JP, Huygen FJ, de Mos M, Martin CG, Sturkenboom MC. Incidence of facial pain in the general population. Pain. 2009;147(1-3):122-127. doi:10.1016/j.pain.2009.08.023

5. Song L, He L, Pei Q, et al. CT-guided percutaneous radiofrequency thermocoagulation for glossopharyngeal neuralgia: a retrospective clinical study of 117 cases. Clin Neurol Neurosurg. 2019; 178:42-45. doi:10.1016/j.clineuro.2019.01.013

6. Blumenfeld A, Nikolskaya G. Glossopharyngeal neuralgia. Curr Pain Headache Rep. 2013;17(7):1-8. doi:10.1007/s11916-013-0343-x

7. Waldman SD. Glossopharyngeal neuralgia. In: Atlas of Uncommon Pain Syndromes. 4 ed. Philadelphia: Elsevier; 2020:62-66.

8. O'Neill F, Nurmikko T, Sommer C. Other facial neuralgias. Cephalalgia. 2017;37(7):658-669. doi:10.1177/0333102417689995

9. Liu Q, Zhong Q, Tang G, He G. Ultrasound-guided glossopharyngeal nerve block via the styloid process for glossopharyngeal neuralgia: a retrospective study. J Pain Res. 2019;12:2503-2510. doi:10.2147/ JPR.S214596

10. Dach F, Éckeli ÁL, Ferreira Kdos S, Speciali JG. Nerve block for the treatment of headaches and cranial neuralgias - a practical approach. Headache. 2015;55(Suppl 1):59-71. doi:10.1111/head.12516

11. Kandan SR, Khan S, Jeyaretna DS, Lhatoo S, Patel NK, Coakham HB. Neuralgia of the glossopharyngeal and vagal nerves: long-term outcome following surgical treatment and literature review. $\mathrm{Br} J$ Neurosurg. 2010;24(4):441-446. doi:10.3109/02688697.2010.487131

12. Ferroli P, Fioravanti A, Schiariti M, et al. Microvascular decompression for glossopharyngeal neuralgia: a long-term retrospective review of the Milan-Bologna experience in 31 consecutive cases. Acta Neurochir (Wien). 2009;151(10):1245-1250. doi:10.1007/s00701009-0330-5

13. Kim MK, Park JS, Ahn YH. Microvascular decompression for glossopharyngeal neuralgia: clinical analyses of 30 cases. $J$ Korean Neurosurg Soc. 2017;60(6):738-748. doi:10.3340/jkns.2017.0506. 010

14. Teixeira MJ, de Siqueira SR, Bor-Seng-Shu E. Glossopharyngeal neuralgia: neurosurgical treatment and differential diagnosis. Acta Neurochir (Wien). 2008;150(5):471-475; discussion 475. doi:10.10 07/s00701-007-1493-6

15. Rey-Dios R, Cohen-Gadol AA. Current neurosurgical management of glossopharyngeal neuralgia and technical nuances for microvascular decompression surgery. Neurosurg Focus. 2013;34(3):E8. doi:10.3171/2012.12.FOCUS12391

16. Uihlein A, Love JG, Corbin KB. Intracranial section of the glossopharyngeal nerve; sensory changes observed postoperatively. AMA Arch Neurol Psychiatry. 1955;74(3):320-324. doi:10.1001/ archneurpsyc.1955.02330150086013

17. Rushton JG, Stevens JC, Miller RH. Glossopharyngeal (vagoglossopharyngeal) neuralgia: a study of 217 cases. Arch Neurol. 1981;38 (4):201-205. doi:10.1001/archneur.1981.00510040027002

18. Lévêque M, Park MC, Melhaoui A, Yomo S, Donnet A, Régis J. Gamma knife radiosurgery for glossopharyngeal neuralgia: marseille experience. J Radiosurg SBRT. 2011;1(1):41-46.

19. Williams BJ, Schlesinger D, Sheehan J. Glossopharyngeal neuralgia treated with gamma knife radiosurgery. World Neurosurg. 2010;73 (4):413-417. doi:10.1016/j.wneu.2010.01.007

20. Wang X, Tang Y, Zeng Y, Ni J. Long-term outcomes of percutaneous radiofrequency thermocoagulation for glossopharyngeal neuralgia: a retrospective observational study. Medicine (Baltimore). 2016;95 (48):e5530. doi:10.1097/MD.0000000000005530

21. Chang MC. Efficacy of pulsed radiofrequency stimulation in patients with peripheral neuropathic pain: a narrative review. Pain Physician. 2018;21(3):E225-e234. 
22. Mollinedo FT, Esteban SL, Vega CG, Orcasitas AC, Maguregi AA. Pulsed radiofrequency treatment in a case of Eagle's syndrome. Pain Pract. 2013;13(5):399-404. doi:10.1111/j.1533-2500.2012.00592.x

23. Chua NH, Beems T, Vissers KC. Two cases of glossopharyngeal neuralgia successfully treated with pulsed radiofrequency treatment. Ann Acad Med Singapore. 2011;40(8):387-389.

24. Shah RV, Racz GB. Pulsed mode radiofrequency lesioning to treat chronic post-tonsillectomy pain (secondary glossopharyngeal neuralgia). Pain Pract. 2003;3(3):232-237. doi:10.1046/j.1533-2500. 2003.03028.x

25. Abejon D, Garcia del Valle S, Nieto C, Delgado C, Gomez-Arnau JI. [Pulsed radiofrequency treatment in idiopathic and secondary glossopharyngeal neuralgia: preliminary results in 2 cases]. Rev Esp Anestesiol Reanim. 2005;52(2):109-114.

26. Bharti N, Chattopadhyay S, Singla N, Bala I, Batra YK, Bakshi J. Pulsed radiofrequency ablation for the treatment of glossopharyngeal neuralgia secondary to oropharyngeal carcinoma. Pain Physician. 2018;21(3):295-302. doi:10.36076/ppj.2018.3.295

27. Headache Classification Committee of the International Headache Society (IHS). The International Classification of Headache Disorders, 3rd edition. Cephalalgia. 2018;38(1):1-211. doi:10.1177/ 0333102417738202

28. Ferreira-Valente MA, Pais-Ribeiro JL, Jensen MP. Validity of four pain intensity rating scales. Pain. 2011;152(10):2399-2404. doi:10.1016/j.pain.2011.07.005

29. Blank FS, Mader TJ, Wolfe J, Keyes M, Kirschner R, Provost D. Adequacy of pain assessment and pain relief and correlation of patient satisfaction in 68 ED fast-track patients. J Emerg Nurs. 2001;27(4):327-334. doi:10.1067/men.2001.116648

30. Landriel Ibañez FA, Hem S, Ajler P, et al. A new classification of complications in neurosurgery. World Neurosurg. 2011;75(5-6):709-715; discussion 604-711. doi:10.1016/j.wneu.2010.11.010

31. Elawamy A, Abdalla EEM, Shehata GA. Effects of pulsed versus conventional versus combined radiofrequency for the treatment of trigeminal neuralgia: a prospective study. Pain Physician. 2017;20 (6):E873-e881.

32. Chen J, Sindou M. Vago-glossopharyngeal neuralgia: a literature review of neurosurgical experience. Acta Neurochir (Wien). 2015;157(2):311-321; discussion 321. doi:10.1007/s00701-0142302-7
33. Luo F, Lu J, Ji N. Treatment of refractory idiopathic supraorbital neuralgia using percutaneous pulsed radiofrequency. Pain Pract. 2018;18(7):871-878. doi:10.1111/papr.12687

34. Luo F, Wang T, Shen Y, Meng L, Lu J, Ji N. High voltage pulsed radiofrequency for the treatment of refractory neuralgia of the infraorbital nerve: a prospective double-blinded randomized controlled study. Pain Physician. 2017;20(4):271-279.

35. Lan M, Zipu J, Ying S, Hao R, Fang L. Efficacy and safety of CT-guided percutaneous pulsed radiofrequency treatment of the Gasserian ganglion in patients with medically intractable idiopathic trigeminal neuralgia. J Pain Res. 2018;11:2877-2885. doi:10.2147/ JPR.S179228

36. Luo F, Meng L, Wang T, Yu X, Shen Y, Ji N. Pulsed radiofrequency treatment for idiopathic trigeminal neuralgia: a retrospective analysis of the causes for ineffective pain relief. Eur J Pain. 2013;17 (8):1189-1192. doi:10.1002/j.1532-2149.2012.00278.x

37. Fang L, Tao W, Jingjing L, Nan J. Comparison of high-voltage with standard-voltage pulsed radiofrequency of gasserian ganglion in the treatment of idiopathic trigeminal neuralgia. Pain Pract. 2015;15 (7):595-603. doi:10.1111/papr.12227

38. Fang H, Zhang J, Yang Y, Ye L, Wang X. Clinical effect and safety of pulsed radiofrequency treatment for pudendal neuralgia: a prospective, randomized controlled clinical trial. J Pain Res. 2018;11:2367-2374. doi:10.2147/JPR.S167866

39. Shi $\mathrm{Y}, \mathrm{Wu} \mathrm{W}$. Treatment of neuropathic pain using pulsed radiofrequency: a meta-analysis. Pain Physician. 2016;19(7):429-444.

40. Giannoni C, White S, Enneking FK, Morey T. Ropivacaine with or without clonidine improves pediatric tonsillectomy pain. Arch Otolaryngol Head Neck Surg. 2001;127(10):1265-1270. doi:10.10 01/archotol.127.10.1265

41. Arias MJ. Percutaneous radio-frequency thermocoagulation with low temperature in the treatment of essential glossopharyngeal neuralgia. Surg Neurol. 1986;25(1):94-96. doi:10.1016/0090-3019(86)90124-2

42. Ori C, Salar G, Giron GP. Cardiovascular and cerebral complications during glossopharyngeal nerve thermocoagulation. Anaesthesia. 1985;40(5):433-437. doi:10.1111/j.1365-2044.1985.tb10843.x

43. Ren H, Shen Y, Luo F. Treatment of supraorbital neuralgia using ultrasound-guided radiofrequency thermocoagulation of the supraorbital nerve: a retrospective study. J Pain Res. 2020;13:251-259. doi:10.2147/JPR.S228720
Journal of Pain Research

\section{Publish your work in this journal}

The Journal of Pain Research is an international, peer reviewed, open access, online journal that welcomes laboratory and clinical findings in the fields of pain research and the prevention and management of pain. Original research, reviews, symposium reports, hypothesis formation and commentaries are all considered for publication. The manuscript management system is completely online and includes a very quick and fair peer-review system, which is all easy to use. Visit http:// www.dovepress.com/testimonials.php to read real quotes from published authors. 